\title{
Between Expectations and Challenges
}

\section{Recherche scientifique avec données empiriques}

\section{Résumé}

La question de la reconnaissance des certifications délivrées par les MOOC représente une sorte de plafond de verre qui limite leur développement. À côté des investissements de prestige, les établissements d'enseignement supérieur qui souhaitent valoriser cette approche massive comme instrument de formation reconnu se retrouvent confrontés à la difficile mise en œuvre de la reconnaissance de l'évaluation. L'étude a porté sur la caractérisation des différentes modalités d'évaluation avec une ambition de reconnaissance universitaire des MOOC, en se focalisant notamment sur les quelques initiatives qui proposent des solutions susceptibles d'apporter une validation institutionnelle à ce qui est mis en œuvre.

\section{Mots-clés}

MOOC, certification universitaire, validation, apprentissages, reconnaissance

\section{Abstract}

The issue of recognition of qualifications awarded by MOOCs is a sort of glass ceiling holding back their development. Despite significant financial investments, higher education institutions wishing to exploit this massive approach as a recognized training instrument find themselves faced with the difficult implementation of the evaluation recognition. The research examined the characterization of various evaluation methods aiming at developing an academic recognition of MOOCs, focusing in particular on some initiatives that try to offer solutions providing institutional validation to what is implemented.

\section{Keywords}

MOOC, academic certification, validation, learning, recognition 


\section{Introduction}

La multiplication des demandes de « reconnaissance des expériences professionnelles » ainsi que l'arrivée de la validation des acquis de l'expérience (VAE) et le souhait de développer les « partenariats avec les entreprises » conduisent à une « transformation des parcours de formation et de l'accès aux certifications universitaires » (Presse, 2013). Dans le même temps, les certifications sur des plateformes en ligne telles que France université numérique (FUN), Coursera, edX, ou encore FutureLearn se sont multipliées. De la certification universitaire en langues au MOOC avec certification payante, les conditions de validation de la réussite aux cours diffèrent. Cet article présente les résultats d'une étude sur les différents types de certifications accessibles en ligne et sur leurs modalités tant de participation que de validation. Pour cela, nous allons tout d'abord présenter les différents types de certification existants ainsi que leurs modalités d'inscription, coûts et démarches certificatives, puis nous mettrons en lumière les principaux aspects problématiques liés à l'émergence et à la prise en compte ou non des certifications et des MOOC, apparus en 2012 (Cisel et Bruillard, 2012) dans le paysage universitaire.

\section{De la participation à un MOOC à la certification}

On constate, en France, que différents sites mettent à disposition des liens vers des MOOC proposés par des organismes certificateurs, par exemple $M O O C$ Francophone (http://mooc-francophone.com) mais aussi Le portail du numérique dans l'enseignement supérieur (http://sup-numerique.gouv.fr). Le premier concerne les MOOC francophones et propose l'essentiel de l'offre francophone alors que le second est un site gouvernemental qui réunit les différentes plateformes MOOC et leur offre de formation. Mais comment les organismes de formation proposent-ils de répondre aux différents besoins des apprenants, notamment ceux concernant une valorisation du cours dans lequel ils s'engagent? Mettent-ils justement en avant l'aspect « certifica- tion " pour les apprenants? Et si oui, comment certifient-ils les candidats?

\subsection{La certification en éducation, c'est quoi, pour qui, pour quoi?}

Quel que soit le pays, le fonctionnement « ancien régime » ne se souciait pas de faire un lien entre une personne et la place qu'elle occupait sur la base de ses compétences. L'université, notamment, était un instrument de reconnaissance des connaissances classiques qui n'étaient en rien professionnelles. Pour ce qui relevait des professions, les corporations réglaient la question par le passage de grades suivant des exigences et des rites intégrant les apprentis, les compagnons et les maîtres. En France, par exemple, dans notre conception moderne, la collation des grades français - monopole de l'État et confiée aux universités - repose sur le décret de Napoléon $1^{\text {er }}$ du 17 mars 1808 établissant les grades de baccalauréat, licence et doctorat. Le master est venu s'ajouter plus récemment avec la réforme LMD (Décret $n^{\circ} 2002-482,2002$ ). Jusqu'à la réforme de 1973, qui énonce que les diplômes d'études universitaires générales (DEUG) et ensuite les autres diplômes, sont composés de modules, les diplômes étaient constitués de différents certificats. Dans l'arsenal français, on retrouve encore des traces de cette époque avec le certificat de capacité en droit. On obtenait donc son grade en réussissant un certain nombre de certificats. Le terme a disparu pendant un certain temps du lexique universitaire relatif aux diplômes pour être affecté à des formations complémentaires sans lien avec les grades, mais qui constituaient des spécialisations. L'instrument "diplôme d'université » est venu remplacer ces certificats « informels » alors que le terme « certificat » est réapparu officiellement avec le certificat de compétences en langues de l'enseignement supérieur (CLES) ou encore avec le certificat informatique et Internet (C2i). Cependant, l'un et l'autre sont déconnectés des grades, même s'ils peuvent être intégrés dans un cursus et de fait apparaître dans ce que l'on désigne par le terme de supplément au diplôme, c'est-à-dire le descriptif 
de son contenu. Dans le même temps, le processus de Bologne et l'instauration du système licencemaster-doctorat (LMD) a bouleversé les modes de reconnaissance des compétences et des connaissances en lien avec les diplômes avec la mise en place de crédits dits « ECTS » (European Credit Transfer Scale) : 180 pour une licence, 120 de plus pour un master. Il s'agit là de la situation française, mais dans d'autres pays francophones, le terme certificat peut recouvrir une réalité liée à l'obtention de grades. C'est le cas en Belgique, en Suisse ou encore au Canada. Les domaines de connaissances et de compétences se sont ainsi complexifiés et morcelés. L'apparition de cursus spécifiques conjugués avec les technologies a fini par se cristalliser sous l'appellation "formations ouvertes à distance » (FOAD) ou « MOOC » (Karsenti, 2013).

Cela amène fatalement l'établissement universitaire à aborder sous un nouveau jour la question de la reconnaissance des compétences et des connaissances issues de ces nouveaux cursus. L'approche classique de l'université avec des convocations aux examens dans des salles surveillées et des soutenances publiques est perturbée par le foisonnement de candidats aux géographies mondiales. Les plateformes mettant en ligne les MOOC mettent à disposition des moyens d'attester de la réussite à des activités et du suivi de la progression proposée. Cette attestation de suivi est généralement attribuée immédiatement après la finalisation et la réussite du MOOC par le candidat. On a alors assisté à un changement et à la mise en place récente de ce que l'on appelle des certifications (Acquatella, 2016). Et cela a son importance, notamment lorsque l'on sait que parmi les éléments de motivation poussant les candidats à participer aux certifications, on retrouve « la reconnaissance universitaire» (Mocquet, Santi, Ammari et Marchand, 2016).

Bien plus que de simples attestations de réussite, ces certifications permettent à l'apprenant de prouver qu'il a suivi un cursus selon des modalités particulières et d'attester de son bon parcours, allant parfois même jusqu'à l'acquisition de crédits universitaires. Mais cette certification officielle amène à une différence importante. En effet, les cours sont toujours proposés librement et gratuitement, mais, lorsque l'on souhaite obtenir une certification officielle, c'est-à-dire garantie par l'université créatrice du cours, une participation financière est bien souvent demandée.

Aussi, si ces formations sont elles aussi diplômantes, quelle est la différence entre les cours à distance sur ces plateformes et les cours traditionnels? Ceux-ci sont-ils basés sur le même fonctionnement (cours et devoirs) ou adoptent-ils une autre posture? Si ce n'est pas le cas, on se rapproche alors de la massification en tant que « véritable effet de nouveauté », comme l'a résumé Minichiello (2013). Y a-t-il alors d'autres perspectives pour ces MOOC au-delà de cette "massification » des formations? Notre question centrale est donc la suivante : De quelle manière se produit ce que l'on appelle la certification, autrement dit, la reconnaissance universitaire, à partir de dispositifs issus des technologies de l'information et de la communication en formation? En d'autres termes : comment les participants à des formations en ligne, de type MOOC, voient-ils la validation de leur participation, et potentiellement de leur réussite, par les organismes certificateurs?

\subsection{Méthodologie d'analyse}

Pour aborder ces différents points, nous allons tout d'abord procéder à une analyse des différentes plateformes qui proposent des cours en ligne accessibles à tous. Nous proposons donc pour cela de mettre en place une méthodologie d'analyse des dispositifs de certification proposés sur les plateformes de cours de type MOOC (par exemple, FUN, Coursera, etc.), mais aussi dans les universités proposant des certifications, en nous inspirant des travaux de Belleflamme et Jacqmin (Belleflamme et Jacqmin, 2014, p. 2). Ces auteurs établissent une typologie des MOOC mettant en avant différents éléments d'analyse. Parmi ceux-ci, on retrouve les questionnements majeurs qui guident leur travail : Quel est le public visé? Quelles sont les activités proposées? Quel est le degré de contrainte pour l'étudiant? Comment l'apprentissage est-il « coté » 
et « certifié »? Le tableau 1 présente la grille d'analyse, inspirée des travaux de Belleflamme et Jacqmin (2014), que nous utiliserons pour étudier les MOOC existants :

\section{Tableau 1}

Grille d'analyse des M00C inspirée des travaux de Belleflamme et Jacqmin (2014)

\begin{tabular}{|l|l|l|}
\hline Caractéristiques de la certification & \multicolumn{1}{|c|}{ Éléments à intégrer } & \multicolumn{1}{c|}{ Exemple } \\
\hline Formation & Intitulé de la formation & $\begin{array}{l}\text { "Comprendre l'écologie, pour } \\
\text { une économie innovante » }\end{array}$ \\
\hline Champ disciplinaire & Champ disciplinaire concerné & Écologie \\
\hline Éditeur & Plateforme et organisme & Coursera - ESCP Europe \\
\hline Type de cours & Cours d'introduction ou cours spécialisés & Cours d'introduction \\
\hline Activités proposées & Types d'activités & Devoir + questionnaire \\
\hline Degré de contrainte & Charge de travail & 2h/semaine \\
\hline Durée & Temps de la formation & 5 semaines \\
\hline Type de validation & Certification payante, gratuite, surveillée & Certificat payant \\
\hline Coût & Prix demandé pour la validation & 44 euros \\
\hline
\end{tabular}

Nous avons ainsi étudié différentes plateformes MOOC et analysé chacun des cours dans le but de collecter les informations que nous venons de préciser. Nous nous sommes par ailleurs inscrits sur ces plateformes afin d'obtenir les informations nécessaires à notre recherche et qui n'étaient pas intuitivement affichées par l'organisme créateur du MOOC ou la plateforme qui le proposait. Cette recherche a été effectuée en 2016 et a permis le recensement de près de $200 \mathrm{MOOC}$ et autres certifications universitaires. Nous avons également procédé à une entrevue avec le président de l'Université de Cergy-Pontoise (France) afin d'obtenir son point de vue sur la question de la certification universitaire.

\section{Principaux résultats de la recherche}

Les données collectées concernent différentes plateformes françaises et européennes. Elles nous ont permis de mettre en évidence les modalités de validation des certifications et leurs différences, mais aussi d'évaluer les conditions de certification de telles formations.

\subsection{Les différentes modalités de validation des certifications}

On constate tout d'abord que les certifications proposées ne comportent pas toutes les mêmes méthodes de validation de la réussite au parcours. On peut ainsi relever des certifications avec ou sans modalité de surveillance. Et cela semble notamment dépendre de la délivrance ou non de crédits universitaires. Nous mettons par ailleurs en évi- 
dence trois modèles majeurs de validation pour les certifications universitaires (tableau 2).

\section{Tableau 2}

Modèles de surveillance et de reconnaissance des certifications

\begin{tabular}{|c|c|c|c|}
\hline $\begin{array}{c}\text { Modalités } \\
\text { d'évaluation et de } \\
\text { validation des cours }\end{array}$ & $\begin{array}{c}\text { Participation et } \\
\text { validation sans } \\
\text { modalité de } \\
\text { surveillance }\end{array}$ & $\begin{array}{c}\text { Participation et validation } \\
\text { avec modalité de } \\
\text { surveillance à distance }\end{array}$ & $\begin{array}{c}\text { Participation et } \\
\text { validation dans des } \\
\text { centres d'examen agréés }\end{array}$ \\
\hline Lieu & À distance & À distance & En présentiel \\
\hline Surveillance & Aucune surveillance & Surveillance & Surveillance \\
\hline Contrôle d'identité & $\begin{array}{c}\text { Pas de contrôle d'identité } \\
\text { des participants }\end{array}$ & $\begin{array}{c}\text { Contrôle d'identité } \\
\text { Contrôle d'identité }\end{array}$ \\
\hline Moyen utilisé & Non & ProctorU' & Humain \\
\hline $\begin{array}{c}\text { Utilisation de locaux } \\
\text { Reconnaissance de } \\
\text { crédits ECTS }\end{array}$ & Non & Non & $\begin{array}{c}\text { Oui, dans les locaux agréés } \\
\text { par l'organisateur }\end{array}$ \\
\hline
\end{tabular}

\subsection{Des dispositifs de certification qui diffèrent d'une plateforme à l'autre}

En ce qui concerne les dispositifs de certification, on constate des distinctions majeures entre les différentes plateformes de formation. Par exemple, la plateforme FUN (http://fun-mooc.fr) propose 184 cours, dont 10 cours certifiants contre paiement, soit $5,43 \%$ des cours proposés. Seuls trois cours permettent l'obtention de crédits universitaires, soit 1,63\% des cours. Sur la plateforme Coursera (http://fr.coursera.org), il n'est jamais fait état de crédits universitaires (à l'exception des cours en partenariat avec l'AUF et donnés par l'EPFL). Sur OpenClassrooms, lorsqu'il s'agit de l'obtention de crédits universitaires à la suite d'un cours, on constate que $17,69 \%$ des cours proposés sont certifiants et que seulement $0,7 \%$ de l'ensemble des cours proposés permettent l'obtention de crédits universitaires (4 cours sur 537). 


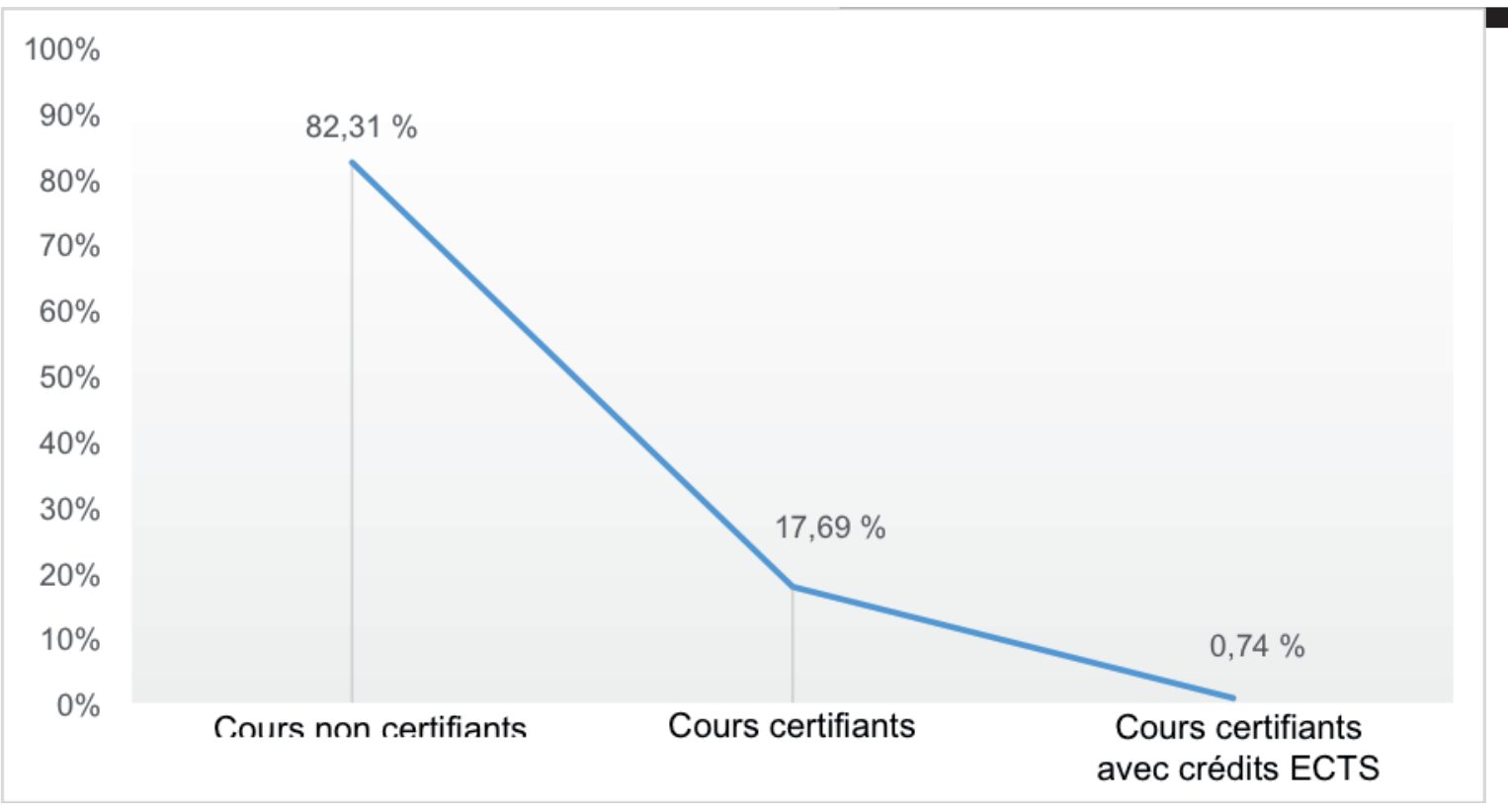

\section{Figure 1}

Répartition des cours chez OpenClassrooms selon le type de certification

En ce qui concerne les plateformes MOOC européennes, sur 152 cours mis en ligne sur FutureLearn (http://futurelearn.com), 148 proposent une certification. La plateforme a lancé, en 2016, les premiers MOOC avec reconnaissance de crédits universitaires, ce qui n'est pas le cas pour la plateforme espagnole Miriadax (http://miriadax.net/ home). Enfin, nous avons pu constater que l'Agence universitaire de la francophonie (AUF) ${ }^{1}$ appuie 32 MOOC actuellement, dont 9 permettent d'obtenir une certification (ce qui représente $28,1 \%$ des MOOC proposés). Les crédits universitaires vont alors de 1 à 3 ECTS pour un montant allant de $40 €$ à $100 €$.

1 Voir la section FOAD MOOC du site de l'Agence (http:// foad-mooc.auf.org).

\subsection{Une nécessaire adaptation de l'offre universitaire en matière de certification}

La formation tout au long de la vie prenant de plus en plus d'ampleur au sein même des universités, il s'agit pour ces établissements de proposer un plan de formation lié à cette nouvelle demande d'éducation permanente. On observe ainsi le projet de l'Université de Cergy-Pontoise qui va passer de 400 millions d'euros de chiffre d'affaires aujourd'hui à 1 milliard d'ici 2020 (Galbaud et Lewandowski, 2016). Pour le président de cette université, François Germinet, il s'agit d'une nécessité pour les universités que «d'apparaître comme des acteurs crédibles, pour ne pas dire indispensables, de la formation tout au long de la vie » (Galbaud et Lewandowski, 2016). Selon lui, la formation continue est aujourd'hui un «besoin pour l'ensemble de la société ». On se dirige donc vers la mise en place de ce type de dispositifs, mais le chemin est encore long et se pose la question du modèle éco- 
nomique à suivre pour ancrer ces dispositifs dans le paysage universitaire. C'est d'ailleurs « leur intégration à des cursus d'établissements supérieurs » qui serait garante de leur réussite (Delpeyroux et Bachelet, 2015). Ces auteurs ont mené une expérimentation dans laquelle des étudiants ont participé à un MOOC (ABC Gestion de projet) dans le cadre de leur cursus universitaire et ont conclu à l'importance de la prise en compte de la réussite du MOOC dans leurs notes dans la mesure où cela leur permettait de « valider une matière/module ou UE ». L'adaptation entre cours ouverts et massifs et cours universitaires semble être un axe prioritaire majeur à prendre aujourd'hui en compte. En effet, il va désormais falloir s'assurer que ces formations sont viables économiquement et, comme le rappelle le président de l'Université de Cergy-Pontoise, il sera nécessaire de "facturer les prestations au coût réel ». L'autre aspect mis en avant est celui de la validation de ces cours par une certification reconnue au niveau universitaire. Aussi, comme le rappelle Harden (2013), le MIT a été la première université à permettre l'obtention d'une certification professionnellement attractive. Selon l'auteur, il s'agissait de «l'un des derniers obstacles » à franchir pour généraliser ce type d'enseignement. Ces certifications deviennent ainsi de plus en plus reconnues et il faut aller vers une validation des certifications par la délivrance de crédits universitaires, comme c'est le cas dans quelques cours que nous avons observés, notamment ceux que propose l'AUF. Le constat de Cisel et Bruillard (2012) ne semble pas avoir grandement évolué depuis quatre ans et la reconnaissance des MOOC en matière de crédits universitaires semble toujours être cantonnée « à quelques formations atypiques ». Pourtant, l'enseignement universitaire évolue et les établissements d'enseignement supérieur se doivent de s'adapter à ce mouvement, comme le confirme François Germinet : "Au-delà des certifications en langues et en informatique, déjà présentes au sein des établissements, le principe de la certification représente un enjeu pour la formation tout au long de la vie. » Dans la mesure où « les formations universitaires se déclinent aujourd'hui de plus en plus en blocs de compétences », proposer une « certification de formations courtes s'appuyant sur ces blocs de compétences représente dès lors un axe de développement très important pour les universités ». On est donc dans une réelle volonté de développement des certifications dans les universités et de leur reconnaissance institutionnelle. Cette reconnaissance est même facilitée en Europe alors qu'elle est plus complexe en Amérique où l'interrogation se pose sur une confusion qui pourrait naître de l'accréditation pour la reconnaissance des $\mathrm{MOOC}$ « en matière de diplomation dans l'enseignement supérieur ». Le système européen des ECTS permet quant à lui d'évacuer cette contrainte par une unification de « la reconnaissance des crédits entre les pays de la communauté européenne» (Grégoire, 2016). Les conditions semblent ainsi optimales pour une union entre certifications et diplômes universitaires en Europe. Pourtant, certaines caractéristiques des MOOC restent contraignantes et pourraient ralentir leur développement. C'est le cas notamment de la reconnaissance de l'évaluation et de la validation des MOOC.

\subsection{Les MOOC, des outils certifiants mais aux modalités d'évaluation et de validation à revoir}

Qu'elles soient universitaires, en langues, en informatique, proposées par Cisco ou encore LPI, on parle de certifications en permanence, mais l'utilisation de ce terme ne semble pas encore clairement définie. Aussi, nous pourrions définir la certification comme une validation du parcours d'un apprenant dans un cursus, quel qu'il soit, et cela sur la base de critères, définis par l'organisme certificateur, à remplir pour attester d'un tel succès. La certification garantirait, finalement, la réussite d'un apprenant dans un parcours d'apprentissage. Les MOOC viennent ainsi encore ajouter à la confusion avec des cours permettant la délivrance d'attestations de participation et de réussite, de badges, mais aussi de plus en plus de certifications pouvant mener à l'obtention de crédits universitaires. Mais ces MOOC peuvent-ils alors prendre la place des dispositifs de certification actuels? Peuvent-ils les 
compléter? Quel bilan faire aujourd'hui depuis leur mise en place et à quoi faut-il s'attendre dans les années à venir? L'émergence des MOOC en 2012 et leur mise en place correspondent à une innovation radicale « dans la manière de mettre en œuvre l'enseignement au niveau de l'enseignement supérieur » (Charlier, 2014) et qui va dans le sens d'un développement de la certification reconnue au grand public. Ces cours d'un nouveau genre cherchent à répondre à un besoin : « s'adresser à un très grand nombre d'étudiants » (Charlier, 2014) avec la possibilité de répondre aux besoins des étudiants d'Afrique subsaharienne " très nombreux et manquant d'accès aux ressources pour apprendre ». Ce type de dispositif permettrait-il de résoudre de telles difficultés? Et comme l'avance l'auteure : « [L]es MOOC ne seraient-ils pas une occasion à ne pas manquer de se centrer davantage sur la qualité de l'enseignement à l'université et sur les missions de l'université de demain? » D'autant plus que les apprenants mettent en valeur les certificats attestant de leur acquisition de connaissances et de compétences sur leurs blogues. Si leur réussite se rapprochait des attentes universitaires, cela ne ferait qu'appuyer leur pertinence aux yeux des participants. Cette relation, justement, entre MOOC et université n'est pas si éloignée, comme le confirme Landry (Landry, 2014) : «N'est-ce pas la mission (nouvelle?) de l'université d'accompagner les jeunes puis les adultes dans leur quête de savoirs et de compétences tout au long de leur vie dans un monde en mutation de plus en plus rapide? » Pourtant, la première constatation par rapport à l'exploitation des MOOC est critique. En effet, ces MOOC ne répondent pas pour le moment à tous les espoirs qu'ils suscitent et on constate des difficultés à leur intégration dans le monde de la formation, notamment en ce qui concerne les interactions entre les participants (enseignants-étudiants et étudiants entre eux). Ils sont qualifiés de "moins riches en ligne que dans un environnement conventionnel» (Belleflamme et Jacqmin, 2014) et ils restent difficilement acceptés en tant que "programmes d'études complets et reconnus ». Selon ces mêmes auteurs, cet écart toujours perceptible entre MOOC et dispositifs traditionnels pourrait se réduire au vu de l'apparition continue de nouvelles technologies plus «adéquates » et de l'émergence de «bonnes pratiques pédagogiques basées sur des fondamentaux solides » (Belleflamme et Jacqmin, 2014, p. 3). D'autres critiques accompagnent le développement des MOOC, notamment le fait que les établissements ne délivrent pas, ou peu, de véritables crédits ni de diplômes (Beck, 2015), ce qui va dans le sens de la critique de Belleflamme et Jacqmin (Belleflamme et Jacqmin, 2014). Aussi, nous avons pu constater qu'à l'exception de l'AUF, qui semble encourager cette mise en relation entre les MOOC et les universités, les plateformes proposant des MOOC tendent peu vers ce modèle. Pendant que l'AUF propose des évaluations surveillées en présentiel pour les certifications, d'autres continuent à proposer des évaluations effectuées à distance sur ProctorU. Est-ce ici le frein à leur insertion? Comme le rappelle Depover (Depover, 2014), « la certification des connaissances dans un MOOC n'est pas sans poser problème, en raison de la difficulté d'identifier avec certitude l'étudiant qui se présente pour compléter une épreuve ». Pour que la valeur universitaire de ces crédits ECTS soit reconnue, il est indispensable qu'ils soient validés par un examen surveillé, au même titre que ceux qui sont proposés dans le cadre des cursus universitaires classiques. La proposition ProctorU ne semble ainsi pas répondre à cette demande et seules les épreuves surveillées sur table sont susceptibles d'attester officiellement de la réussite du candidat. Pour que la validation de certifications soit officielle, il apparaît indispensable de développer encore les dispositifs d'examens sur table, comme le fait l'AUF. En effet, la surveillance à distance ne garantit aucunement la validité et l'égalité entre les candidats. Personne ne pourra vérifier totalement l'environnement de l'apprenant lors de son examen. Cisel et Bruillard (Cisel et Bruillard, 2012) confirment cette crainte et cette inadéquation entre dispositif mis en place et attentes universitaires: "La première critique contre la valeur de ces certifications vient du fait que, de par leur nature même, rien ne garantit que la personne qui passe l'examen en ligne est bien la personne dont le nom est inscrit sur le certificat ». 
L'avenir des MOOC et autres cours certifiants réside peut-être justement, et surtout, dans cette reconnaissance de leur modèle d'évaluation et de validation, d'autant plus que l'étude des tendances typologiques des candidats à certaines certifications confirme cette quête de reconnaissance universitaire (Mocquet et al., 2016). Cette dernière se doit donc d'être des plus justifiées et équitables.

\subsection{Un modèle économique à définir au plus vite}

Le rapport de France Stratégie (Harden, 2013) confirme également une recherche par les établissements universitaires de l'intégration de ces MOOC dans leurs offres de formation. Mais le modèle économique le permettant semble difficile à trouver et à mettre en place pour les établissements, notamment ceux du public. Ceux-ci pourraient pourtant « délocaliser leurs cours grâce aux MOOC », « toucher plus d'étudiants avec moins d'enseignants » et faire des économies non négligeables. Les possibilités existent, reste à trouver le modèle à appliquer pour pérenniser ces cours sans mettre en difficulté les universités. Ce modèle économique à suivre pourrait être celui du «freemium, c'est-à-dire la combinaison de services gratuits et payants » (Delpech et Diagne, 2016), comme c'est le cas sur de nombreuses plateformes que nous avons étudiées offrant un cours partiellement gratuit mais une certification payante (tableau 3). En d'autres termes, on peut accéder à certains composants gratuits du cours, mais il est nécessaire d'acheter le cours pour obtenir une certification officielle de réussite.

\section{Tableau 3}

Exemples de cours dont une partie du contenu est gratuite mais où la certification est payante

\begin{tabular}{|c|c|c|}
\hline Nom & Domaine & $\begin{array}{l}\text { Plateforme } \\
\text { — Éditeur }\end{array}$ \\
\hline $\begin{array}{l}\text { Créer et développer une } \\
\text { startup technologique }\end{array}$ & Entrepreneuriat & Coursera - HEC \\
\hline $\begin{array}{l}\text { Programmation et } \\
\text { politiques financières } \\
\text { — partie } 1\end{array}$ & Finance & $e d X-F M I$ \\
\hline $\begin{array}{l}\text { Psychologie de } \\
\text { la négociation }\end{array}$ & Négociation & $e d X-U C L$ \\
\hline $\begin{array}{l}\text { Devenir entrepreneur } \\
\text { du changement }\end{array}$ & Entrepreneuriat & $\begin{array}{l}\text { Coursera - HEC } \\
\text { Paris }\end{array}$ \\
\hline $\begin{array}{l}\text { L'avenir de la décision: } \\
\text { connaître et agir } \\
\text { en complexité }\end{array}$ & Entrepreneuriat & Coursera - ESSEC \\
\hline $\begin{array}{l}\text { Espace mondial, une } \\
\text { vision française des } \\
\text { global studies }\end{array}$ & Politique & $\begin{array}{l}\text { Coursera - } \\
\text { Sciences Po }\end{array}$ \\
\hline $\begin{array}{l}\text { Introduction au } \\
\text { game design }\end{array}$ & Design & IONISx - e-artsup \\
\hline
\end{tabular}

Le rapport de France Stratégie met d'ailleurs en valeur la nécessité pour les plateformes d'« européaniser » leur offre et de ne pas se cantonner à des cours nationaux. D'autant plus que les plateformes européennes ne proposent que $2 \%$ de MOOC internationaux alors que Coursera, plateforme américaine de cours, en propose $70 \%$ qui font partie du « top 50 » du classement de Shanghai. D'un point de vue économique, ce rapport pointe le faible taux de certification des différentes plateformes $(8 \%$ pour edX) et le besoin de « diversifier leurs sources de revenus ». Aussi, certains établissements qui proposent l'attribution de crédits ECTS le font en permettant à leurs étudiants d'acquérir ces crédits immédiatement ou à ceux qui n'y sont pas inscrits de les obtenir en intégrant leur école. Ce dispositif permettrait donc d'amener l'apprenant vers l'université certificatrice et, potentiellement, de le recruter dans les années qui suivent cette certification, dans la mesure où il bénéficiera déjà de cer- 
tains crédits ECTS. Cette dernière correspondrait donc ici à un appel à l'inscription universitaire et au prérecrutement des établissements en offrant, avant toute inscription, des crédits à de potentiels futurs étudiants. Cette hypothèse est d'ailleurs en relation avec l'objectif premier des MOOC, à savoir que «même si plusieurs MOOC semblent, à première vue, gratuits, il s'agit souvent de stratégies - un peu comme celles qu'utilisent certaines grandes entreprises commerciales pour attirer les clients potentiels : des étudiants » (Karsenti, 2013). Et si l'objectif des MOOC n'était pas uniquement de proposer un contenu gratuit à des apprenants en soif de savoir mais bel et bien de recruter des étudiants pour réaliser davantage de bénéfices? Ou est-ce une question de survie des universités? À ce moment-là, si les certifications ou MOOC devenaient payants, il apparaîtrait inévitable qu'en contrepartie, les établissements fournisseurs s'assurent que l'apprenant repart avec un cours universitaire et une certification de même niveau. Reste alors à garantir l'égalité d'accès et de validation de ces cours à tous. Une autre problématique liée au développement de ces certifications dans le monde universitaire actuel semble résider ici.

\section{Conclusion}

Les universités font aujourd'hui face à une multiplication des cours proposés, notamment ceux qui sont offerts à distance. En dehors des cursus traditionnels, les établissements universitaires proposent des certifications qui peuvent porter sur les langues ou les compétences informatiques. L'émergence des MOOC a amené un certain nombre d'établissements à repenser le schéma traditionnel de validation des compétences, notamment par la certification. Cette certification peut dans certains cas permettre l'obtention de crédits ECTS reconnus par l'établissement universitaire. En proposant des certifications permettant l'obtention de ce type de crédits, les universités peuvent non seulement mettre en place un prérecrutement d'étudiants et satisfaire le besoin des apprenants en quête de reconnaissance officielle par l'Université, mais aussi s'installer en tant qu'acteurs majeurs de la forma- tion tout au long de la vie. On constate pourtant que ces certifications permettant l'obtention de crédits ECTS restent rares. Un des freins éventuels à cette lente mise en place des certifications repose peutêtre sur les conditions de validation qui sont proposées pour juger de la réussite ou de l'échec des candidats aux épreuves à réaliser. En effet, très peu de certifications sont validées en présentiel avec vérification d'identité et devoir sur table. Pourtant, cela devrait être une condition indispensable à la reconnaissance officielle des crédits ECTS proposés. Alors que les certifications en informatique et en langues, depuis longtemps mises en place par les universités, sont validées par un examen en présentiel, les certifications issues des MOOC le sont très peu. Certains établissements font plutôt appel à des applications de vérification telles que ProctorU pour laquelle la validité de la surveillance mise en place peut prêter à discussion et à contestation.

Ainsi, si les universités veulent réellement développer ces certifications tout au long de la vie en les faisant reconnaître par la délivrance de crédits ECTS, et donc par une reconnaissance universitaire, elles se doivent de repenser la validation de ces crédits acquis, comme le fait l'AUF, par exemple, en proposant une évaluation finale sur table et surveillée. L'avenir des certifications universitaires passe par leur validité sur le plan tant du contenu que de la modalité de validation. Enfin, il est indispensable de repenser le statut ou la modalité des certifications universitaires, aussi bien dans leur mise en place par les universités elles-mêmes que dans leur pérennisation grâce à un modèle économique viable.

\section{Références}

Acquatella, F. (2016). Le COOC, un autre visage du MOOC. Distances et médiations des savoirs, 2016(14). https://doi.org/10.4000/dms.1386

Beck, S. (2015, 3 juin). Les MOOC: des savoirs «accessibles à tous»? Récupéré le 18 avril 2016 du site du projet ADJECTIF : http://www.adjectif.net 
Belleflamme, P. et Jacqmin, J. (2014). Les plateformes MOOCs. Menaces et opportunités pour l'enseignement universitaire. Regards Economiques, 110. Récupéré de l'archive ORBi de l'Université de Liège : http://orbi.ulg.ac.be

Charlier, B. (2014). Les MOOC: une innovation à analyser. Distances et médiations des savoirs, 2014(5). Récupéré de http://journals.openedition.org/dms

Cisel, M. et Bruillard, E. (2012). Chronique des MOOC. STICEF, 19. Récupéré de http://sticef.univ-lemans.fr

Décret n²002-482 du 8 avril 2002 portant application au système français d'enseignement supérieur de la construction de l'Espace européen de l'enseignement supérieur (2002). Récupéré du site Légifrance : http://legifrance.gouv.fr

Delpech, Q. et Diagne, M. (2016). MOOC, l'âge de maturité? Modèles économiques et évolutions pédagogiques (document de travail no 2016-01). Récupéré du site France stratégie : http://strategie.gouv.fr

Delpeyroux, S. et Bachelet, R. (2015). Intégrer un MOOC dans un cursus de formation initiale. Dans Actes $d u 8^{e}$ colloque Questions de pédagogie dans l'enseignement supérieur (QPES 2015) (p. 61-69). Récupéré du site du colloque : http://colloque-pedagogie.org

Depover, C. (2014). Quels modèles économiques et pédagogiques pour les MOOC? Distances et médiations des savoirs, 2014(5). Récupéré de http://journals.openedition.org/dms

Galbaud, D. et Lewandowski, J.-C. (2016, 28 mars). Formation continue: «Les universités doivent apparaittre comme des acteurs crédibles». Le Monde Campus. Récupéré de http://lemonde.fr

Grégoire, R. (2016). Cours en ligne ouverts et massifs : états des lieux et adoption au Canada français. Guide et bilan de l'impact des cours en ligne ouverts et massifs (CLOM) au Canada francophone. Récupéré du site du REFAD : http://refad.ca

Harden, N. (2013). La fin de l'Université que nous connaissions. Commentaire, (142), 359-368. doi:10.3917/ comm.142.0359

Karsenti, T. (2013). Les MOOC, révolution ou simple effet de mode? Revue internationale des technologies en pédagogie universitaire, $10(2)$. https://doi.org/10.18162/ritpu.2013.227

Landry, P. (2014). Les MOOC changeront-ils le rôle des universités? Distances et médiations des savoirs, 2014(6). Récupéré de http://journals.openedition.org/dms
Minichiello, F. (2013). Le phénomène des MOOCs (Massive Open Online Courses). Revue internationale d'éducation de Sèvres, 2013(63), 12-16.

https://doi.org/10.4000/ries.3453

Mocquet, B., Santi, C., Ammari, I. et Marchand, T. (2016). De l'inscription à un MOOC à la certification académique: Le cas de la collection de MOOC «compétences du numérique et $\mathrm{C} 2 \mathrm{i}$ Niveau 1». Revue internationale des technologies en pédagogie universitaire, 13(1), 47-63. https://doi.org/10.18162/ritpu-2016-v13n1-04

Presse, M.-C. (2013). La prise en compte de l'expérience dans un dispositif de formation: Tensions et stratégies organisationnelles. Questions vives, recherches en éducation, 10, 63-76.

https://doi.org/10.4000/questionsvives.1378

\section{Note}

1 ProctorU (http://proctoru.com) est une entreprise d'évaluation à distance. 